\title{
Basic fibroblast growth factor in an animal model of spontaneous mammary tumor progression
}

\author{
STEVEN KAO, JEFFREY MO, ANDREW BAIRD and BRIAN P. ELICEIRI \\ Department of Surgery, School of Medicine, University of California San Diego, MC 8236, San Diego, CA 92103, USA
}

Received December 31, 2011; Accepted February 6, 2012

DOI: $10.3892 /$ or.2012.1710

\begin{abstract}
Although basic fibroblast growth factor (FGF2) was the first pro-angiogenic molecule discovered, it has numerous activities on the growth and differentiation of non-vascular cell types. FGF2 is both stimulatory and inhibitory, depending on the cell type evaluated, the experimental design used and the context in which it is tested. Here, we investigated the effects of manipulating endogenous FGF2 on the development of mammary cancer to determine whether its endogenous contribution in vivo is pro- or anti-tumorigenic. Specifically, we examined the effects of FGF2 gene dosing in a cross between a spontaneous breast tumor model $\left(\mathrm{PyVT}^{+}\right.$ mice) and $\mathrm{FGF}^{-/-}$(FGF KO) mice. Using these mice, the onset and progression of mammary tumors was determined. As predicted, female FGF2 WT mice developed mammary tumors starting around 60 days after birth and by 80 days, $100 \%$ of FGF2 WT female mice had mammary tumors. In contrast, $80 \%$ of FGF2 KO female mice had no palpable tumors until nearly three weeks later (85 days) at times when $100 \%$ of the WT cohort was tumor positive. All FGF KO mice were tumor-bearing by 115 days. When we compared the onset of mammary tumor development and the tumor progression curves between FGF het and FGF KO mice, we observed a difference, which suggested a gene dosing effect. Analysis of the tumors demonstrated that there were significant differences in tumor size depending on FGF2 status. The delay in tumor onset supports a functional role for FGF2 in mammary tumor progression, but argues against an essential role for FGF2 in overall mammary tumor progression.
\end{abstract}

\section{Introduction}

The prevalence of breast cancer remains a significant clinical problem, where xenograft models using established tumor cell lines have limitations for the study of mechanisms regulating tumor onset (1). In contrast, transgenic models

Correspondence to: Dr Brian P. Eliceiri, Department of Surgery, University of California San Diego, 212 Dickinson Street, MC 8236, San Diego, CA 92103, USA

E-mail: beliceiri@ucsd.edu

Key words: breast cancer, bFGF, angiogenesis such as the polyomavirus middle-T oncogene (PyVT) mouse model driven by the mouse mammary tumor virus (MMTV) promoter, have been shown to be a reliable and comparable mouse model of breast tumor onset and progression (2-4). The steps of spontaneous mouse mammary tumor progression in this model parallel the histological progression in human breast cancer $(3,5-7)$.

Breast and mammary tissues have been shown to have extraordinary plasticity during adult life and are thought to depend, at least in part, on inhibitory interactions between mammary myoepithelial cells (MECs) and luminal breast epithelial cells (BECs) (8). Indeed, these same interactions are thought to be important in normal development and function of the mammary gland $(8,9)$. Several years ago, investigators proposed that a loss of inhibitory processes $(2,10)$ might disinhibit positive feedback loops thereby allowing for tumor progression (11). One candidate is FGF2, which controls epithelial function at the MEC and BEC interface and acts as a survival and differentiation factor (12). While low levels of FGF2 in breast cancer are associated with a poor prognosis and response to treatment $(13,14)$ there is evidence that FGF2 overexpression results in enhanced tumor growth due to increased angiogenesis (15). This discrepancy may highlight that FGF2 signaling can potentially have a dual role of suppressing and promoting tumor growth.

We investigated the possibility that breast cancer progression may be driven in part by a disinhibition of BEC that results when FGF2 expression at the MEC-BEC interface is lost. The progression of spontaneous mammary tumors in the transgenic MMTV-PyVT mouse model $\left(\mathrm{PyVT}^{+/+}\right)$is a multistep process (2) that involves many of the cell targets of FGF2 (5,16-18). Because the myoepithelium is a natural endogenous source of FGF2 in mammary tissue (12), we hypothesized that an absence of FGF2 might affect the onset and progression of mammary tumor development in $\mathrm{PyVT}^{+}$mice. In the event that endogenous FGF2 might exert competing effects on tumor progression through inhibition of epithelial cells at the MEC-BEC interface, or alternatively due to its angiogenic effects, we evaluated its activity in a model of spontaneous tumor development.

\section{Materials and methods}

Mice. All animal studies were conducted with the approval of, and under the oversight of the Institutional Animal Care and 

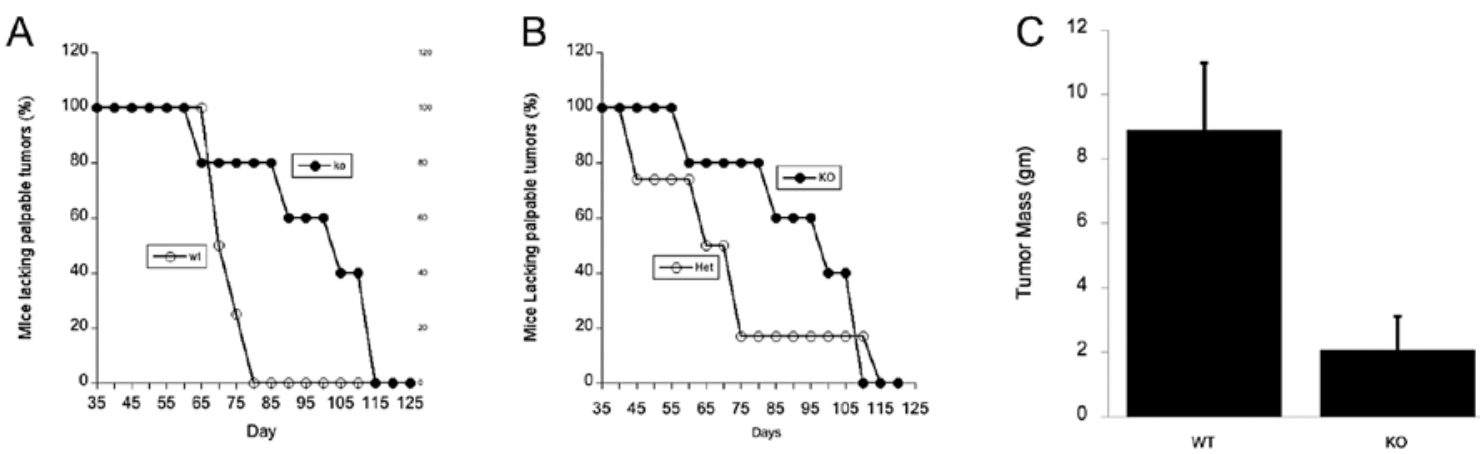

Figure 1. Delayed progression of tumorigenesis and decreased tumor size in knockout and heterozygous mice for FGF2 as compared to normal mice. (A) Tumor onset of tumor-bearing PyVT ${ }^{+}$FGF2 KO and WT mice (P<0.05). (B) Tumor onset of tumor-bearing PyVT ${ }^{+}$FGF2 het vs. WT mice (P<0.05). (C) Mammary tumor wet weight in tumor bearing WT and FGF2 KO mice upon harvest at $110-125$ days $(\mathrm{P}<0.05)$.

Use Committee of the University of California, San Diego. We bred FGF $^{-/}$SV129/Black Swiss female mice with transgenic hemizygous MMTV-PyVT FVB male mice $\left(\mathrm{PyVT}^{+}\right)$for three generations to yield $\mathrm{FGF}^{-/}$; $\mathrm{PyVT}^{+}(\mathrm{FGF} 2 \mathrm{KO}), \mathrm{FGF}^{+/}$; $\mathrm{PyVT}^{+}$(FGF2 het), and $\mathrm{FGF}^{+/+}$; $\mathrm{PyVT}^{+}$(WT). We validated that the tumor onset of the $\mathrm{FGF}^{+/+}$was consistent with the 60-80 day onset described in previous reports (6). Tissue expression studies were conducted using tissue harvested from mice that were collected after cervical dislocation. Genotypes were identified from tail DNA by PCR using PCR primers specific for PyVT and FGF2.

Tumor measurements. Cohorts of female WT, FGF2 het, and FGF2 KO mice were followed to evaluate mammary tumor onset, incidence, growth and progression. After weaning, body weights of the mice were recorded weekly and the presence of palpable lesions in the mammary glands was determined. Following excessive weight loss or the presence of tumors in excess of $15 \mathrm{~mm}$ in width or length from caliper measurements, the mice were sacrificed. Tumor volumes at various time points and tumor weights at necropsy were compared between the three groups using a Wilcoxon-Rank test.

Antibodies. To demonstrate FGF2 immunoreactivity, a polyclonal antibody was raised in rabbits by immunization against peptides 1-24 of bovine FGF2. This antibody has high affinity for extracellular FGF2 and cross-reactivity with FGF2 from several species, as previously described $(19,20)$. Antibodies for Factor VIII and FGFR1 were obtained from Biocare Inc. (Concord, CA, USA) and Sigma-Aldrich (St. Louis, MO, USA), respectively.

Immunohistochemistry. Mouse mammary fat pads (MFP) were obtained following euthanasia of animals, perfusion with PBS and fixation with 4\% paraformaldehyde (PFA) in phosphate-buffered saline (PBS), pH 7.4. Formalin-fixed lung specimens were stored in paraffin by UCSD Histology Core Services. At the time of immunohistological staining, paraffin sections were first deparaffinized in xylene and in progressively more dilute aqueous solutions of ethanol. Sections were then incubated with Proteinase K (Millipore cat\#21627 $0.2 \mathrm{mg} / \mathrm{ml}$ ) for $10 \mathrm{~min}$ at room temperature.
Sections were blocked with normal goat serum (ABC Rabbit Kit PK-4002) in PBS for $1 \mathrm{~h}$ and incubated with primary antibody for Factor VIII (Biocare Inc.) antibody or FGF2 and FGFR1 (Sigma-Aldrich) at concentrations of 1:100, 1:4000 and 1:2000 overnight at $4^{\circ} \mathrm{C}$ in $1 \%$ bovine serum albumin in PBS. Sections were washed and incubated with biotin-conjugated secondary antibody (ABC Rabbit Kit PK-4002) for $30 \mathrm{~min}$ at room temperature. Sections were washed again and incubated in $0.3 \% \mathrm{H}_{2} \mathrm{O}_{2}$ to quench endogenous peroxidase activity for $20 \mathrm{~min}$ before the sections were treated with an avidin biotin complex (ABC) (Vectastatin, Burlingame, CA) for amplification of signal. Sections were incubated with diaminobenzidine substrate for $15 \mathrm{~min}$ and after washing, were counterstained with hematoxylin and dehydrated. The coverslips were mounted with VectaMount Mounting Solution and imaged with an Olympus FXS100-BSW microscope.

Quantification of vessel density. In order to quantitatively determine the effect of FGF knockout on blood vessel density, 3 representative fields each measuring about $1500 \mu \mathrm{m}$ by $1000 \mu \mathrm{m}$ on slides stained during the same experiment were analyzed. Blood vessels, as highlighted by IHC for Factor VIII, and confirmed by their morphology, were counted in samples from normal mice, mice lacking one, and mice lacking both copies of FGF2. Average vessel counts from the three groups (each $n=3$ ) were then compared. Error bars represent the standard error of the mean (SEM).

\section{Results}

Delayed progression of tumorigenesis and decreased tumor size in FGF2 knockout and heterozygous mice. The development of tumors in normal mice (WT) and mice lacking one (FGF2 het) or both copies of FGF2 (FGF2 KO) (Fig. 1A and $\mathrm{B}$ ) was marked by significant differences in kinetics. Data collected during mammary development showed that FGF KO mice had delayed onset of tumorigenesis. WT mice began to have palpable tumors by day 65 , and by day 80 all mice in the cohort had tumors. In contrast, while some FGF2 $\mathrm{KO}$ mice began to have palpable tumors around the same time frame, most FGF2-KO mice showed a significantly delayed progression of tumorigenesis, with some palpable 

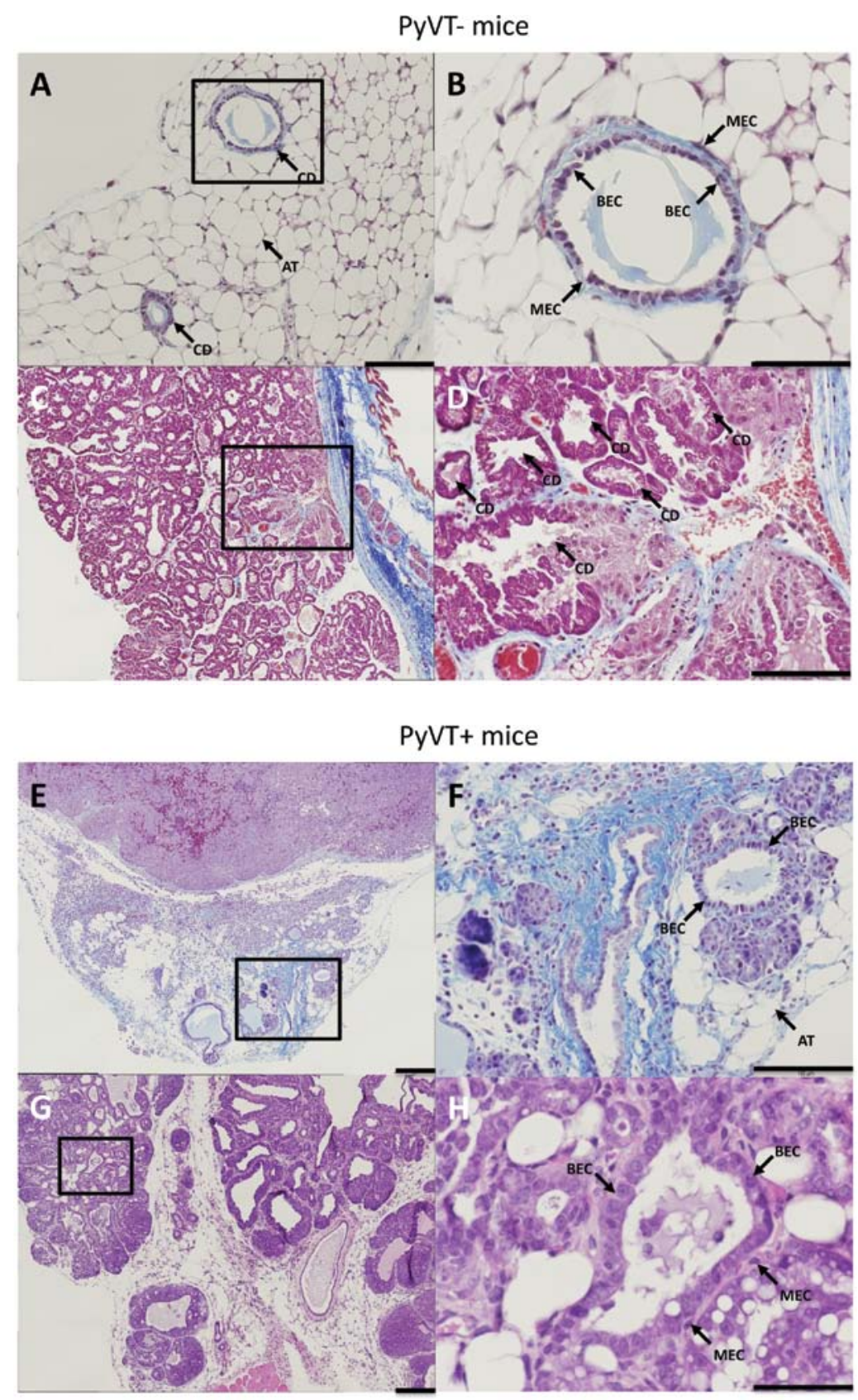

Figure 2. Ductal morphology in normal (PyVT) mammary gland is comparable to those in PyVT ${ }^{+}$mice. (A) Masson's trichrome staining of mammary gland from normal PyVT mouse at 10 weeks of age, with collecting ducts (CD) and adipose tissue (AT) indicated. (B) High magnification of inset from panel A with myoepithelial cells (MEC) and luminal breast epithelial cells (BEC) are indicated. (C) Masson's trichrome staining of mammary gland prepared from a normal lactating mouse at 26 weeks of age. (D) High magnification of inset from panel C. (E) Masson's trichrome staining of mammary gland from PyVT mouse at 22 weeks of age. (F) High magnification of inset from panel E. (G) H\&E staining of mammary gland from FGF2 KO mouse at 14 weeks of age. (H) High magnification of inset from panel G. Scale bars, $200 \mu \mathrm{m}$ (C, E and G); $100 \mu \mathrm{m}$ (A, D, F and H); $50 \mu \mathrm{m}$ (B).

tumors only appearing around day 110. Similarly, FGF2 het mice showed an intermediate phenotype and the onset of tumorigenesis was earlier than was seen in FGF2 KO mice, but later than was seen in WT. This was important to note because it provided evidence of a gene dosing effect and a decreased expression, rather than only a complete knockout affecting tumor growth. Mammary tumors (Fig. 1C) in FGF2 KO mice were also significantly smaller $(\mathrm{P}<0.05)$ than those in WT mice $(8 \mathrm{~g}$ in WT mice vs. $2 \mathrm{~g}$ in, FGF2 KO mice). The significant difference in tumor burden indicated that FGF2 KO mouse tumor cells grew more slowly and/or die at a higher rate than those in WT mice tumors and was consistent with the data presented earlier indicating delayed onset tumorigenesis.

Ductal morphology of normal mice mammary gland is comparable to that observed in PyVT mice. To further characterize mammary tumor development in the absence of FGF2, we performed histological characterization of primary tumors and of mammary fat pads at various stages in development. Masson's trichrome (MT) staining of mammary gland highlighted ductal morphology in normal mouse at 10 (Fig. 2A and B) and 26 (Fig. 2C and D) weeks of age. MT staining in defined ductal structures showed a clear 

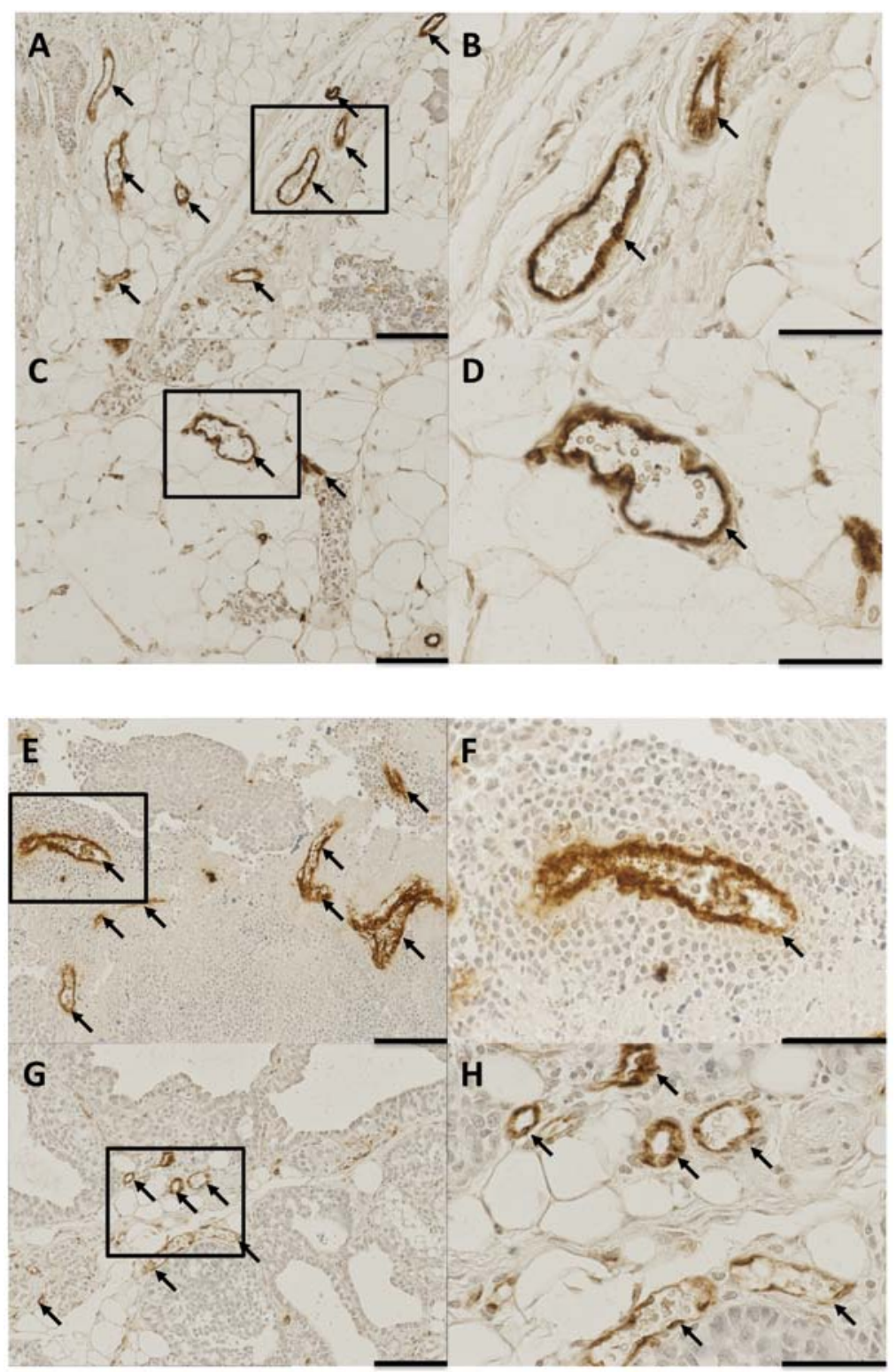

I

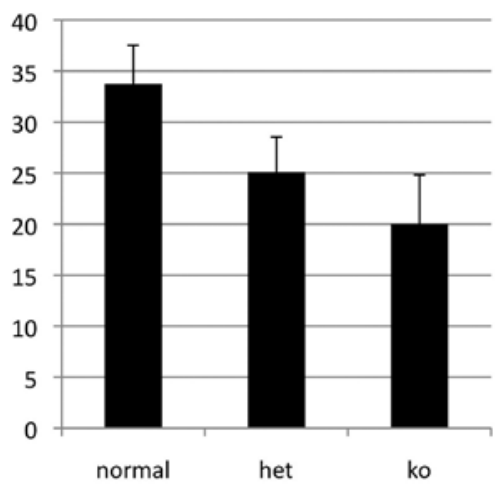

delineation of myoepithelial (MECs) and basal epithelial cells (BECs) separated by basement membrane. Similar ductal structures (Fig. 2E-H) were seen in tumor-bearing WT mice in regions surrounding the tumor mass (Fig. 2E) as well as in areas of interspersed tumor foci (Fig. 2G). Thus, the introduction of the PyVT oncogene did not change the gross development of normal epithelial structures in young mice but
Figure 3. Loss of FGF2 results in decreased tumor vascularization. (A) Immunolocalization of Factor VIII (arrows) in a normal mouse mammary fat pad at 17 weeks of age. (B) High magnification of inset from panel A. (C) Immunolocalization of Factor VIII in an FGF2 KO mouse mammary fat pad at 19 weeks of age. (D) High magnification of panel C. (E) Immunolocalization of Factor VIII in a WT mouse mammary fat pad at 22 weeks of age. (F) High magnification of inset in panel E. (G) Immunolocalization of Factor VIII in a FGF2 KO mouse mammary fat pad at 14 weeks of age. (H) High magnification of inset in panel G. MEC, myoepithelial cells; BEC, luminal breast epithelial cells; AT, adipose tissue. (A, C, E and G) Scale bars, $100 \mu \mathrm{m}$; (B, D, F and H), $500 \mu \mathrm{m}$. (I) Quantification of blood vessel density decrease in tumor bearing FGF2 KO (n=3), FGF2 het $(\mathrm{n}=3)$ and WT $(\mathrm{n}=4)$ mice. The decrease in vascularization between $\mathrm{WT}$ and FGF2 KO was statistically significant $(\mathrm{P}<0.05)$.

rather, served to promote the remodeling and progression to tumorigenesis of MECs and BECs.

Loss of FGF2 results in decreased MFP vascularization in both normal and PyVT $T^{+}$mice. Immunohistochemistry staining for Factor VIII highlighted the tumor vasculature and enabled quantification of tumor angiogenesis (Fig. 3). 


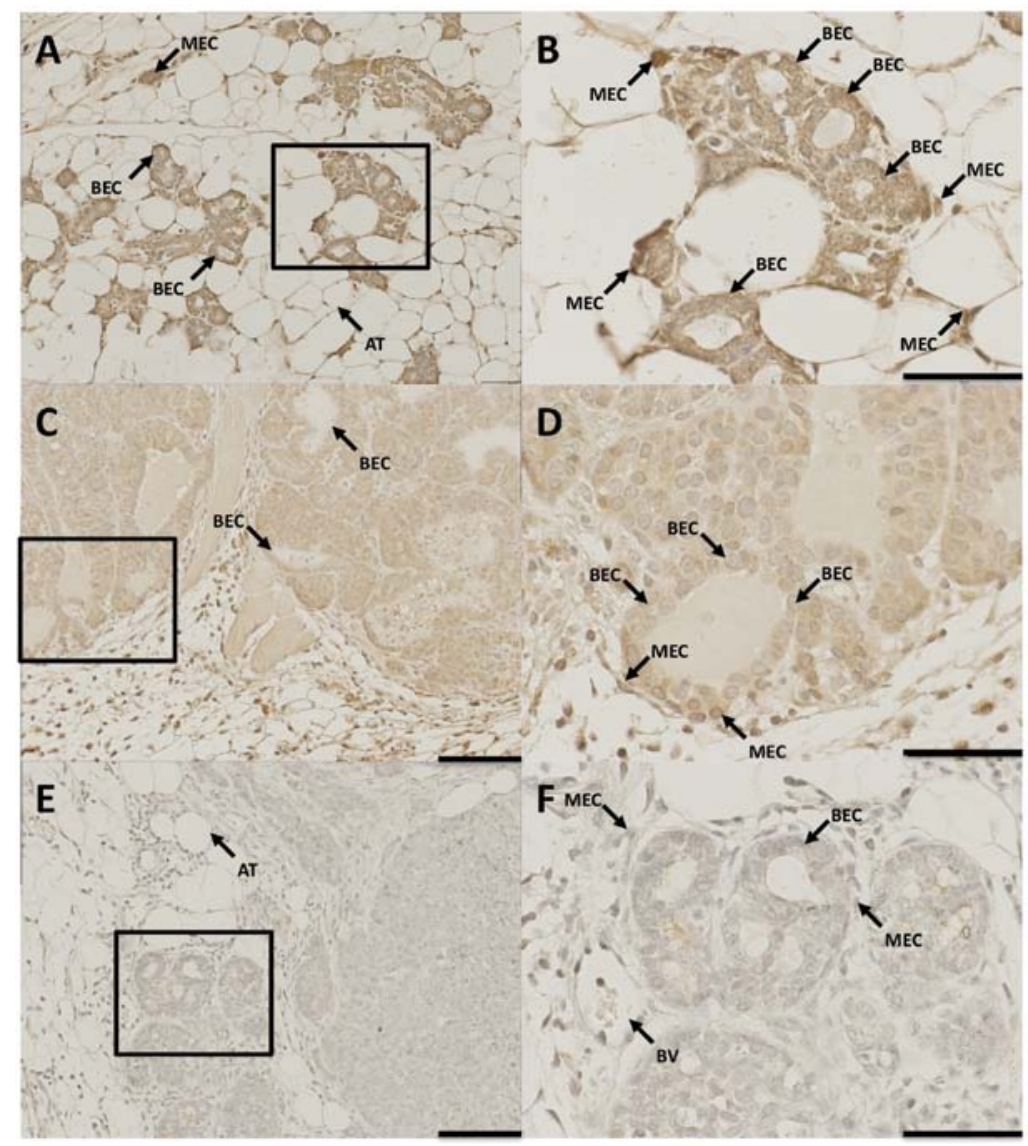

Figure 4. FGF2 present in normal (non-PyVT) and WT while absent in FGF2 KO mice. (A) Immunolocalization of FGF2 in a normal mouse mammary fat pad at 17 weeks of age. (B) High magnification of panel A. (C) Immunolocalization of FGF2 in a WT mouse mammary fat pad. (D) High magnification of inset in panel C. (E) Immunolocalization of FGF2 in FGF2 KO mouse mammary tumor at 19 weeks of age. (F) High magnification of inset in panel E. MEC, myoepithelial cells; BEC, luminal breast epithelial cells; BV, blood vessel; AT, adipose tissue; CD, collecting ducts. Scale bars, $100 \mu \mathrm{m}$ (A, C and E); $50 \mu \mathrm{m}$ $(\mathrm{B}, \mathrm{D}$ and $\mathrm{F})$.

Normal mice (Fig. 3A and B) demonstrated greater vascular density compared to FGF2 KO mice (Fig. 3C and D; arrows) at 17-19 weeks of development. Similarly, tumor-bearing WT mice (Fig. 3E and F; arrows) also showed greater vascular density as compared to FGF2 KO (Fig. 3G and H; arrows). Furthermore, blood vessel density was significantly lower (Fig. 3I) in FGF KO mice mammary tumors when compared to FGF-WT, demonstrating a role for FGF2 in mammary tumor angiogenesis.

FGF2 in mammary tissue of normal, WT and FGF2-KO mice. To characterize the role of FGF2 in normal and tumorbearing mammary tissue, immunohistochemical staining was performed to determine the presence and localization of FGF2 in breast tumor ductal epithelium. Diffuse staining for FGF2 was present throughout the myo- and basal ductal epithelium of normal (non-PyVT ${ }^{+}$) (Fig. 4A and B) and transgenic PyVT mammary tumors (Fig. 4C and D), but absent in FGF2 KO (Fig. 4E and F) mice. The similar staining pattern seen in normal and transgenic PyVT tumors indicated that the introduction of the PyVT oncogene had a limited effect on the expression and localization of endogenous FGF2. In FGF KO tumors, the diffuse staining pattern described earlier was absent, confirming loss of FGF2 immunoreactivity in the knockout.
FGF receptor type 1 (FGFR1) in mammary tissue of normal, $W T$ and FGF2-KO mice. The distribution of FGFR1 was localized to specific ductal morphological structures and demonstrated that there exists a potential pathway for FGF2 activity in mammary tissue. Immunohistochemistry staining confirmed the presence of FGFR1 immunoreactivity in both MECs and BECs in normal (Fig. 5A and B), PyVT ${ }^{+}$and FGF $\mathrm{KO}$ (Fig. 5E and F) mice. The similar staining patterns seen in the three treatment groups show that FGFR1 expression is not significantly influenced by the presence of the PyVT oncogene or the absence of FGF2.

\section{Discussion}

In this study, we assessed whether FGF2 inhibits the onset, growth and progression of mammary tumors or acts as a proangiogenic factor that accelerates tumorigenesis. Specifically, we characterized the expression of FGF2 and FGFR1 in the normal and transgenic mouse mammary glands, and assessed tumor development and progression in FGF2 KO and identified the differences in mammary gland morphology and tumor vascularization. FGF2 and FGFR1 in normal and PyVT-positive mice localized to the myo- and basal epithelium. The data demonstrate that the loss of FGF2 resulted in decreased angiogenesis and a delay in tumor onset. In FGF2 


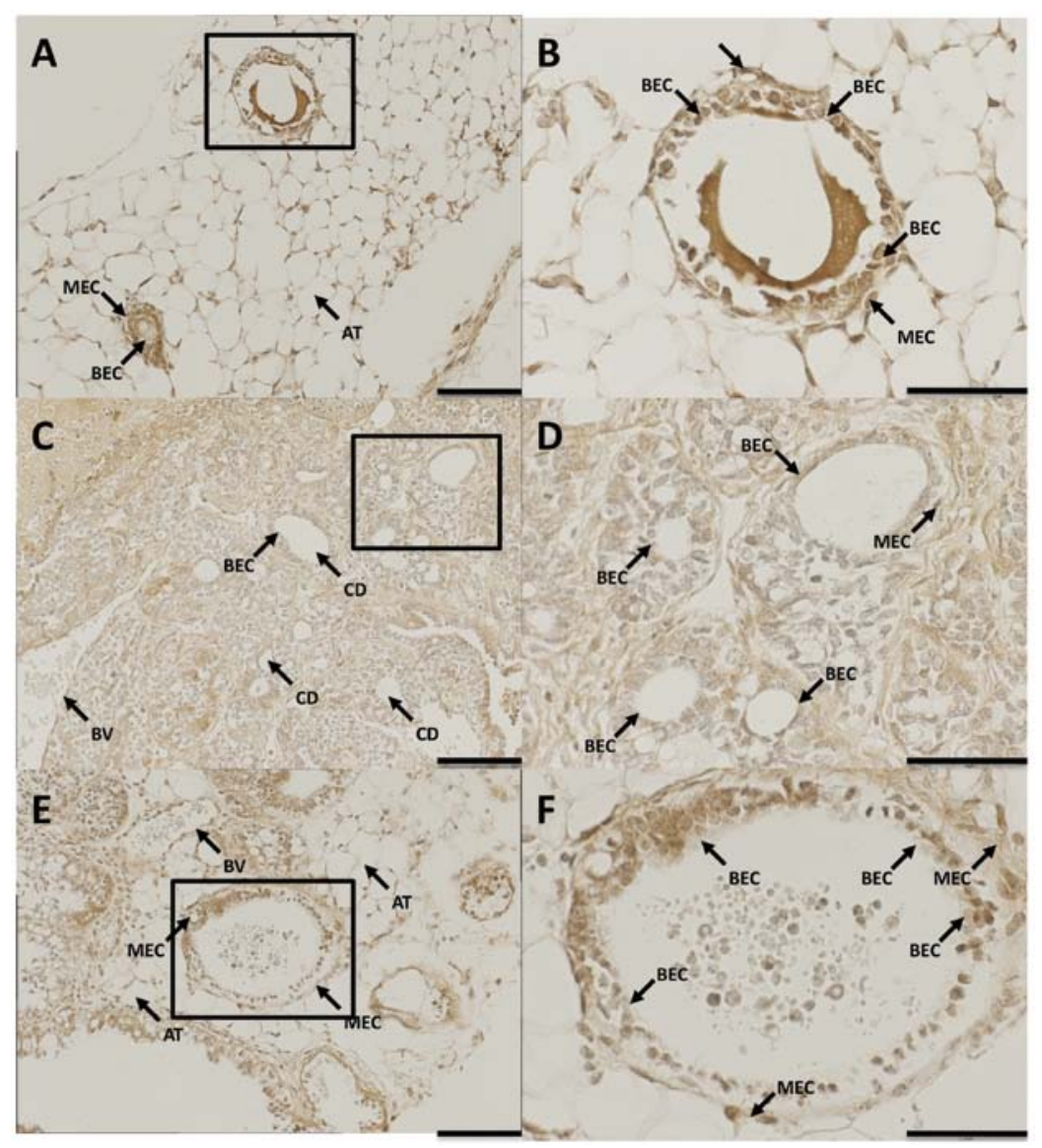

Figure 5. FGF receptor type 1 (FGFR1) present throughout MFPs in WT, and FGF2-KO mice. (A) Immunolocalization of FGFR1 in a normal (non-PyVT) mouse mammary tissue at 10 weeks of age. (B) High magnification of inset from panel A. (C) Immunolocalization of FGFR1 in a WT mouse mammary tumor at 22 weeks of age. (D) High magnification of inset from panel C. (E) Immunolocalization of FGFR1 in a FGF2-KO mouse mammary tumor at 14 weeks of age. (F) High magnification of inset from panel D. MEC, myoepithelial cells; BEC, luminal breast epithelial cells; BV, blood vessel; AT, adipose tissue; CD, collecting ducts. Scale bars, $100 \mu \mathrm{m}$ (A, C and E); $50 \mu \mathrm{m}$ (B, D and F).

KO mice mammary tumors, blood vessel density was markedly reduced with the loss of FGF2 resulting in delayed tumor growth as well as tumors that were significantly smaller. Importantly, mice that were heterozygous for FGF2 showed an intermediate phenotype in tumor onset and growth. This evidence of a gene dosing effect implies that the action of FGF2 depends on its level of expression rather than an 'all-or-none' model of signal transduction. Despite the delay, all of the mice in the cohort eventually developed tumors, suggesting that FGF2 plays at most an ancillary role in tumorigenesis. The results are consistent with the hypothesis that FGF2 contributes to tumor development and the progression to malignancy.

Previous studies implicated FGF2 as a pleiotropic (21) but locally acting cytokine that has distinct juxtacrine roles at the interface been various cell types $(15,22-24)$. One of the many established functional roles of endogenous FGF2 is angiogenesis (25), but it is always exogenous FGF2 that has been shown to stimulate endothelial proliferation and tube formation in in vitro and in vivo models $(26,27)$. The role of FGF2 in cancer angiogenesis has been assumed based on its production by most cancer cell lines $(28,29)$. However, it is important to note that when breast cancer cell lines are evaluated in vitro or in xenograft models to investigate tumor-stroma interactions, the results are often not applicable to in vivo tumorigenesis due to differences in the composition of the local extracellular matrix (30-33) and the absence of various tissue specific stromal interactions (34-36). Furthermore, tumor cells lines are derived from cells at very late stages of tumor progression and selected for growth in cell culture over many passages (37). At the same time, naturally occurring tumors are rare and unpredictable. In order to overcome these difficulties, we used the MMTV-PyVT mouse model. This model has a high frequency of tumor development (5), where the PyVT is under control of the mouse mammary tumor virus long terminal repeat promoter (MMTV-LTR) (6). This model has been shown to be an accurate representation of the development of human breast cancer $(3,4)$. The studies described here provide the in vivo demonstration that loss of FGF2 confers a less malignant phenotype due to reduced vascularization.

Furthermore, FGF2 and its receptors have been shown to be highly expressed in many breast cancers (38) and has been shown to have direct mitogenic effects in the mammary epithelium as well as activation of migration (17). Studies have demonstrated a close interaction between hypoxia and heparin sulfate proteoglycans that modulate the levels of FGF2 signaling (39). However, previous studies described FGF2 as an upstream mediator of angiogenesis (40) that affects further inflammatory responses that are necessary to complete the process of neo-vascularization (18). In light of these data 
as well as the findings presented here, we propose that the action of FGF2, while important in initiating angiogenesis in response to hypoxia-induced stress common in the tumor microenvironment as well as direct mitogenic effects through inhibition of p53, upregulation of survivin (41), and induction of Mdm2 (42), may be superceded by other inflammatory mediators following sufficient inflammatory signaling when it is lost. This hypothesis may harbor significant implications for future therapeutics that attempt to target the action of FGF2. Rather than solely targeting the action of FGF2 on mammary epithelium, there will need to be a combination of therapeutics that will prevent bypassing of FGF2-induced angiogenesis.

There are a variety of clinical studies that have shown that low serum levels of FGF2 correlate with a more malignant phenotype (13), larger tumor size, later disease stage (14), and worse overall and disease-free survival $(13,14)$. In addition, many pre-clinical studies have also shown that overexpression of FGF2 inhibits growth (43), although the exact pathway has yet to be elucidated (44). In contrast, our study shows that the loss of one or both copies of FGF2 results in smaller tumors. Further studies are needed to reconcile this incongruity.

The primary goal of this study was to analyze the contribution of FGF2 to tumor development and progression to malignancy in vivo through gene knockout. Because FGF2 $\mathrm{KO}$ mice were shown to have delayed tumor development and growth compared to PyVT controls with endogenous levels of FGF2, we suggest that FGF2 promotes tumor angiogenesis and progression to malignancy. This experiment has validated the concept that FGF2 blockade in the context of breast cancer can be a beneficial therapeutic intervention. The data obtained here demonstrate that the PyVT mammary tumors are sensitive to the absence of FGF2 and identify the PyVT model as an important preclinical model to evaluate therapies aiming to remove FGF2 function. Our results support the conventional view that, presumably because of its powerful mitogenic activity, FGF2 is a pro-tumorigenic growth factor.

\section{Acknowledgements}

This research was supported by a Department of Defense IDEA grant (AB). We thank Alexandra Borboa for her excellent technical support.

\section{References}

1. Borowsky AD: Choosing a mouse model: experimental biology in context - the utility and limitations of mouse models of breast cancer. Cold Spring Harb Perspect Biol 3: a009670, 2011.

2. Lin EY, Jones JG, Li P, et al: Progression to malignancy in the polyoma middle $\mathrm{T}$ oncoprotein mouse breast cancer mode provides a reliable model for human diseases. Am J Pathol 163: 2113-2126, 2003.

3. Maglione JE, Moghanaki D, Young LJ, et al: Transgenic Polyoma middle-T mice model premalignant mammary disease. Cancer Res 61: 8298-8305, 2001.

4. Qiu TH, Chandramouli GV, Hunter KW, Alkharouf NW, Green JE and Liu ET: Global expression profiling identifies signatures of tumor virulence in MMTV-PyMT-transgenic mice: correlation to human disease. Cancer Res 64: 5973-5981, 2004.

5. Kaplan DR, Pallas DC, Morgan W, Schaffhausen B and Roberts TM: Mechanisms of transformation by polyoma virus middle T antigen. Biochim Biophys Acta 948: 345-364, 1989.
6. Guy CT, Cardiff RD and Muller WJ: Induction of mammary tumors by expression of polyomavirus middle $\mathrm{T}$ oncogene: a transgenic mouse model for metastatic disease. Mol Cell Biol 12: 954-961, 1992.

7. Bautch VL, Toda S, Hassell JA and Hanahan D: Endothelial cell tumors develop in transgenic mice carrying polyoma virus middle T oncogene. Cell 51: 529-537, 1987.

8. Gudjonsson T, Adriance MC, Sternlicht MD, Petersen OW and Bissell MJ: Myoepithelial cells: their origin and function in breast morphogenesis and neoplasia. J Mammary Gland Biol Neoplasia 10: 261-272, 2005.

9. Sternlicht MD, Kedeshian P, Shao ZM, Safarians S and Barsky SH: The human myoepithelial cell is a natural tumor suppressor. Clin Cancer Res 3: 1949-1958, 1997.

10. Barsky SH and Karlin NJ: Myoepithelial cells: autocrine and paracrine suppressors of breast cancer progression. J Mammary Gland Biol Neoplasia 10: 249-260, 2005.

11. Rentsch CA, Cecchini MG and Thalmann GN: Loss of inhibition over master pathways of bone mass regulation results in osteosclerotic bone metastases in prostate cancer. Swiss Med Wkly 139: 220-225, 2009.

12. Gomm JJ, Browne PJ, Coope RC, et al: A paracrine role for myoepithelial cell-derived FGF2 in the normal human breast. Exp Cell Res 234: 165-173, 1997

13. Yiangou C, Gomm JJ, Coope RC, et al: Fibroblast growth factor 2 in breast cancer: occurrence and prognostic significance. $\mathrm{Br} \mathrm{J}$ Cancer 75: 28-33, 1997.

14. Colomer R, Aparicio J, Montero S, Guzman C, Larrodera L and Cortes-Funes H: Low levels of basic fibroblast growth factor (bFGF) are associated with a poor prognosis in human breast carcinoma. Br J Cancer 76: 1215-1220, 1997.

15. Okunieff P, Fenton BM, Zhang L, et al: Fibroblast growth factors (FGFS) increase breast tumor growth rate, metastases, blood flow, and oxygenation without significant change in vascular density. Adv Exp Med Biol 530: 593-601, 2003.

16. Boilly B, Vercoutter-Edouart AS, Hondermarck H, Nurcombe V and Le Bourhis X: FGF signals for cell proliferation and migration through different pathways. Cytokine Growth Factor Rev 11: 295-302, 2000.

17. Vercoutter-Edouart A, Lemoine J, Smart CE, et al: The mitogenic signaling pathway for fibroblast growth factor-2 involves the tyrosine phosphorylation of cyclin D2 in MCF-7 human breast cancer cells. FEBS Lett 478: 209-215, 2000.

18. Andres G, Leali D, Mitola S, et al: A pro-inflammatory signature mediates FGF2-induced angiogenesis. J Cell Mol Med 13: 2083-2108, 2009.

19. Gonzalez AM, Taylor WM, Johanson CE, et al: Co-localization and regulation of basic fibroblast growth factor and arginine vasopressin in neuroendocrine cells of the rat and human brain. Cerebrospinal Fluid Res 7: 13, 2010.

20. Gonzalez AM, Buscaglia M, Ong M and Baird A: Distribution of basic fibroblast growth factor in the 18-day rat fetus: localization in the basement membranes of diverse tissues. J Cell Biol 110: 753-765, 1990.

21. Xian W, Schwertfeger KL, Vargo-Gogola T and Rosen JM: Pleiotropic effects of FGFR1 on cell proliferation, survival, and migration in a 3D mammary epithelial cell model. J Cell Biol 171: 663-673, 2005.

22. Baird A: Fibroblast growth factors: what's in a name? Endocrinology 132: 487-488, 1993.

23. Nugent MA and Iozzo RV: Fibroblast growth factor-2. Int $\mathrm{J}$ Biochem Cell Biol 32: 115-120, 2000.

24. Ornitz DM and Itoh N: Fibroblast growth factors. Genome Biol 2: Reviews3005, 2001

25. Kerbel RS: Tumor angiogenesis: past, present and the near future. Carcinogenesis 21: 505-515, 2000.

26. Folkman J and Klagsbrun M: Angiogenic factors. Science 235: 442-447, 1987.

27. Passaniti A, Taylor RM, Pili R, et al: A simple, quantitative method for assessing angiogenesis and antiangiogenic agents using reconstituted basement membrane, heparin, and fibroblast growth factor. Lab Invest 67: 519-528, 1992.

28. Moscatelli D, Presta M, Joseph-Silverstein J and Rifkin DB: Both normal and tumor cells produce basic fibroblast growth factor. J Cell Physiol 129: 273-276, 1986.

29. Ueba T, Mori H, Takahashi JA, Nozaki K and Hashimoto N: A dyad symmetry element in the fibroblast growth factor- 2 gene promoter with different levels of activity in astrocytoma and hepatocelluar carcinoma cell lines. J Neurooncol 78: 107-111, 2006. 
30. van Kempen LC, de Visser KE and Coussens LM: Inflammation, proteases and cancer. Eur J Cancer 42: 728-734, 2006.

31. Elenbaas B and Weinberg RA: Heterotypic signaling between epithelial tumor cells and fibroblasts in carcinoma formation. Exp Cell Res 264: 169-184, 2001.

32. Demaria F, Porcher R, Sheik-Ismael S, Amarenco G and Benifla JL: Recording expulsive forces during childbirth using intercostal muscle electromyogram: a pilot study. Gynecol Obstet Fertil 33: 299-303, 2005 (In French).

33. Littlepage LE, Egeblad M and Werb Z: Coevolution of cancer and stromal cellular responses. Cancer Cell 7: 499-500, 2005.

34. Tlsty TD and Hein PW: Know thy neighbor: stromal cells can contribute oncogenic signals. Curr Opin Genet Dev 11: 54-59, 2001.

35. Bhowmick NA, Neilson EG and Moses HL: Stromal fibroblasts in cancer initiation and progression. Nature 432: 332-337, 2004.

36. Radisky D, Hagios C and Bissell MJ: Tumors are unique organs defined by abnormal signaling and context. Semin Cancer Biol 11: 87-95, 2001.

37. Burdall SE, Hanby AM, Lansdown MR and Speirs V: Breast cancer cell lines: friend or foe? Breast Cancer Res 5: 89-95, 2003

38. Blanckaert VD, Hebbar M, Louchez MM, Vilain MO, Schelling ME and Peyrat JP: Basic fibroblast growth factor receptors and their prognostic value in human breast cancer. Clin Cancer Res 4: 2939-2947, 1998.
39. Khurana A, Liu P, Mellone P, et al: HSulf-1 modulates FGF2and hypoxia-mediated migration and invasion of breast cancer cells. Cancer Res 71: 2152-2161, 2011.

40. Schumacher B, von Specht BU, Haberstroh J and Pecher P The stimulation of neo-angiogenesis in the ischemic heart by the human growth factor FGF. J Cardiovasc Surg 39: 445-453, 1998.

41. Teh SH, Hill AK, Foley DA, McDermott EW, O'Higgins NJ and Young LS: COX inhibitors modulate bFGF-induced cell survival in MCF-7 breast cancer cells. J Cell Biochem 91: 796-807, 2004.

42. Shaulian E, Resnitzky D, Shifman O, et al: Induction of Mdm2 and enhancement of cell survival by bFGF. Oncogene 15: 2717-2725, 1997.

43. Maloof $\mathrm{P}$, Wang $\mathrm{Q}$, Wang $\mathrm{H}$, et al: Overexpression of basic fibroblast growth factor (FGF-2) downregulates Bcl-2 and promotes apoptosis in MCF-7 human breast cancer cells. Breast Cancer Res Treat 56: 153-167, 1999.

44. Wieder R, Fenig E, Wang H, et al: Overexpression of basic fibroblast growth factor in MCF-7 human breast cancer cells: lack of correlation between inhibition of cell growth and MAP kinase activation. J Cell Physiol 177: 411-425, 1998. 